\title{
Ultra-Mini Percutaneous Nephrolithotripsy
}

Janak Desai, MS, MCh

Department of Urology, Samved Hospital, Ahmedabad, India.

E-mail: drjanakddesai@gmail.com

Ronak Solanki, MS

Department of Urology, Samved Hospital, Ahmedabad, India.

(c) Mary Ann Liebert, Inc. DOI: 10.1089/vid.2013.0013
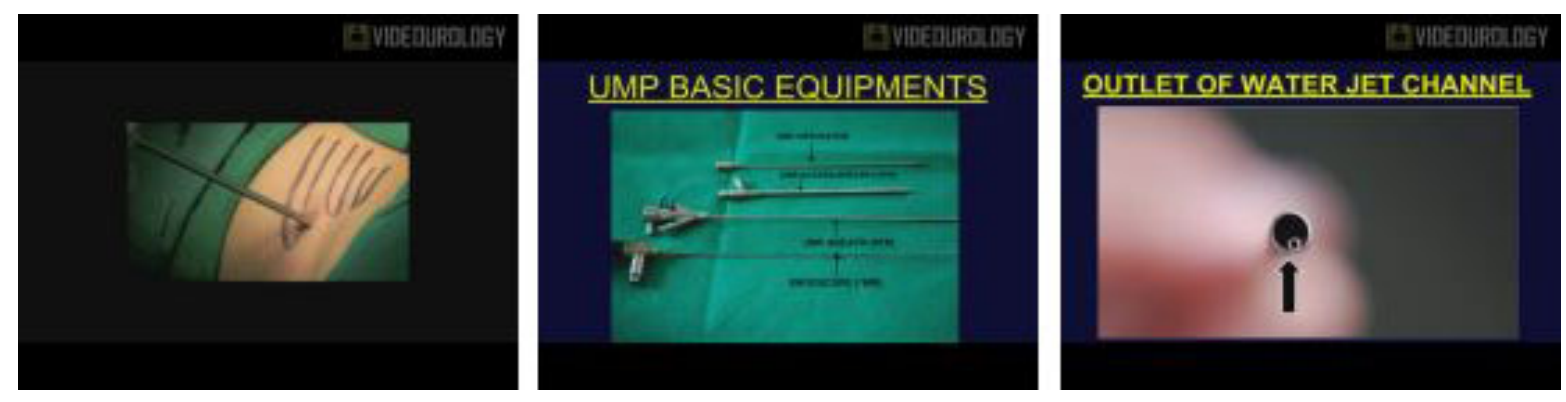

\section{Abstract}

Introduction and Objectives: We want to introduce a new technique for removing medium bulk renal stones. The parenchymal damage is less if the dilation is less, ${ }^{1,2}$ and therefore, we dilate up to $11 \mathrm{~F}$ and use a sheath of $11 \mathrm{~F}$ only. The design of the sheath is the invention and we want to present the design and the outcome because of that design. We disintegrate the stone with a $365 \mu \mathrm{m}$ Holmium laser fiber and keep the fragment size to $2 \mathrm{~mm}$ approximately. At the end, we create a whirlpool effect (an eddy of water) by injecting saline through a very small side channel-the fragments come out automatically.

Methods: A standard puncture is done to access the preferred calyx. Over the guide wire, an $8 \mathrm{~F}$ teflon dilator is used, and then a $11 \mathrm{~F}$ dilation is done. ${ }^{3}$ The Ultra-Mini percutaneous nephrolithotripsy (UMP) sheath is introduced in the pelvicalyceal system. The telescope used is a $3.5 \mathrm{~F}$ slender telescope, which has a 17000 pixel resolution (Schoelly-LUT). The stone is visualized and disintegration is done with a laser fiber. The UMP sheath has a special design (patent pending) and this helps us to create a turbulence in the pelvicalyceal system. The fragments are pulled into the whirlpool effect and are extracted automatically into the sheath and finally expelled outside. ${ }^{4}$

Results: We have done 108 cases so far. This includes four cases of solitary kidney, three cases of failed retrograde intra-renal surgery (RIRS), four cases of failed shock wave lithotripsy (SWL), two cases of obesity, one case of horseshoe kidney, one case of ectopic kidney, one case of chronic renal failure, and two pediatric cases. No case of serious bleeding was reported. We had to convert two UMP into Mini-PNL, because of bleeding that obscured vision. One patient developed hydrothorax because of upper calyx puncture on left side. No case of septicemia was reported. The average stone size was $17.4 \mathrm{~cm}$. The stonefree rate was $86.11 \%$ (93 patients were stone free-confirmed by the CT-Scan done after 30 days).

Conclusions: UMP is a new technique on the horizon. It can remove medium size renal stone (preferable in the lower calyx, pediatric patients, solitary kidneys etc.) with minimal damage to the parenchyma. The use of disposables is much less than RIRS and the learning curve is minimal for surgeons doing PNL regularly. No need of placing a double-J stent or a nephrostomy tube. The patient can be discharged within 12 to 24 hours after surgery. This is a new technique that can be very helpful in select patients, but further comparison with available modalities are required to establish its role in the management of medium size renal calculi.

No competing financial interests exist.

Runtime of video: 7 mins 47 secs 


\section{Cite this video}

Janak Desai, Ronak Solanki, Ultra-Mini Percutaneous Nephrolithotripsy, J Endourol Part B Videourology. 2013, DOI: 10.1089/vid.2013.0013.

\section{References}

1. Knoll T, Wezel F, Michel MS, et al. Do patients benefit from miniaturized tubeless percutaneous nephrolithotomy? A comparative prospective study. J Endourol 2010;24:1075.

2. Kukreja R, Desai M, Patel S, et al. Factors affecting blood loss during percutaneous nephrolithotomy: prospective study. J Endourol 2004;18:715.

3. Yamaguchi $A$, et al. Operating times and bleeding complications in percutaneous nephrolithotomy: A comparison of tract dilation methods in 5,537 patients in the Clinical Research Office of the Endourological Society Percutaneous Nephrolithotomy Global Study. J Endourol 2011;25:933-939.

4. Acheson DJ. Elementary Fluid Dynamics. Oxford: Clarendon Press, 1990, ISBN 0-19-859679-0.

Original Publication Date: 2013 